\title{
Optimal Design on Robustness of Scale-Free Networks Based on Degree Distribution
}

\author{
Jianhua Zhang, ${ }^{1}$ Shuliang Wang, ${ }^{1}$ and Yixing Wang ${ }^{2,3}$ \\ ${ }^{1}$ School of Electrical Engineering and Automation, Jiangsu Normal University, Xuzhou 221116, China \\ ${ }^{2}$ School of Government, Beijing Normal University, Beijing 100875, China \\ ${ }^{3}$ People's Government of Wuduan Town, Peixian, Xuzhou 221638, China
}

Correspondence should be addressed to Jianhua Zhang; zhangjianhua1980@126.com

Received 3 April 2016; Revised 7 June 2016; Accepted 5 July 2016

Academic Editor: Meng Guo

Copyright (C) 2016 Jianhua Zhang et al. This is an open access article distributed under the Creative Commons Attribution License, which permits unrestricted use, distribution, and reproduction in any medium, provided the original work is properly cited.

\begin{abstract}
This paper uses 2-norm degree and coefficient of variation on degree to analyze the basic characteristics and to discuss the robustness of scale-free networks. And we design two optimal nonlinear mixed integer programming schemes to investigate the optimal robustness and analyze the characteristic parameters of different schemes. In this paper, we can obtain the optimal values of the corresponding parameters of optimal designs, and we find that coefficient of variation is a better measure than 2-norm degree and two-step degree to study the robustness of scale-free networks. Meanwhile, we discover that there is a tradeoff among the robustness, the degree, and the cost of scale-free networks, and we find that when average degree equals 6 , this point is a tradeoff point between the robustness and cost of scale-free networks.
\end{abstract}

\section{Introduction}

Complex networks have become more and more important to our daily life [1-5], such as biology system, electrical power grid, transportation network, and pipeline networks. The security and reliability of these networks have been the concern of more and more scientists. Since the discovery of small-world networks, the small-word property [2] and scale-free property [3] have attracted continuous attention from all over the world. It has been recognized that many networks have scale-free property which means that the degree distribution follows a power-law distribution $[4,5]$.

Recently, there are many novel concepts and approaches in many subjects, such as information science, control science, statistical and nonlinear physics, and mathematics and social science which are used to investigate the characteristics in many fields, especially complex network $[6,7]$. The critical point is that the information flow between topological nodes and other physical quantities is important to network security, so we must keep the information exchange unimpeded. Because of the ubiquity of scale-free networks in natural and manmade systems, the security and reliability of these networks have attracted great interest $[8,9]$. The work by Albert demonstrated that scale-free networks possess the robust yet fragile property and he found that it is robust against random failures of nodes but fragile to intentional attacks [3-5].

Cascading failure can occur in many complex systems; an intuitive thinking suggests that the possibility of breakdown of networks triggered by attacks or failures cannot be ignored in scale-free networks. Avalanche of breakdown is a serious threat to the network when nodes and links are sensitive to overloading. The removal of nodes which resulted from random breakdown or intentional attack can change the balance of flows and lead to redistributing loads all over the network; sometimes the redistribution of loads cannot be tolerated and might trigger a cascade of overload failure [10]; finally the network would be collapsing. But it can also propagate and cut down information transmission of the whole network in some cases [11]. Therefore the robustness is an important aspect to investigate the characteristics of scalefree network.

Based on the statistical property of computer network, it is known that computer network is a scale-free network. 
Supercomputer is a computer with a high-level computational capacity compared to a general-purpose computer. With the developments of supercomputers, supercomputer networks will also be formed in the future; therefore supercomputer networks also possess scale-free property, and the reliability and robustness of supercomputer network become more and more important. The optimal design of supercomputer networks can improve the reliability and robustness of supercomputer networks and also enhance speed of the information transmission and exchange of supercomputer networks. Hence the optimal design can present the theoretical and practical significances for the design and construction of supercomputer networks.

This paper is organized as follows. Section 2 discusses the basic characteristics by analyzing the characteristic parameters of scale-free networks. Two-norm degree and its optimal programming of degree distribution are studied in Section 3. Section 4 investigates the coefficient of variation and its optimal design of degree distribution. Finally, a conclusion is presented in Section 5.

\section{Basic Characteristics of Scale-Free Network}

Recently, many researchers have constructed several schemes to measure the robustness of scale-free networks, such as the average degree, average two-step degree (ATSD) [12], and entropy $[13,14]$. In this paper, we construct two models defined as the average 2-norm degree (ATND) and coefficient of variation $(\mathrm{CV})$ to discuss the robustness of scale-free network. From Motter and Lai [11], we know that the node $v_{i}$ of complex network has a load capacity as follows:

$$
C\left(v_{i}\right)=(1+\lambda) L\left(v_{i}\right), \quad i=1,2, \ldots, N
$$

where $\lambda>0$ is the tolerance parameter, $L\left(v_{i}\right)$ is the initial load of node $v_{i}$, and $N$ is the total number of vertices.

The network is designed as $G=\langle V, E\rangle, V=$ $\left\{v_{1}, v_{2}, \ldots, v_{N}\right\}$ is the set of vertices, and $E \subseteq V \times V$ is the set of edges which can exchange information from vertices to others. According to $[12,13,15]$, one knows that the robustness can be improved with the increase of the heterogeneity, and the larger the average degree is, the better the robustness is. Many complex networks are scale-free networks, and the degree distribution of nodes is power-law distribution [3-5], the density function being as follows:

$$
f(k) \sim c k^{-\beta}, \quad \beta \in(2,3) .
$$

Based on the discrete property of degree and continuous approximation principle, we can declare

$$
\begin{aligned}
1 & =\sum_{k=m}^{M} c k^{-\beta}=c \sum_{k=m}^{M} k^{-\beta} \approx c \int_{m}^{M} k^{-\beta} d k \\
& =c \frac{M^{1-\beta}-m^{1-\beta}}{1-\beta},
\end{aligned}
$$

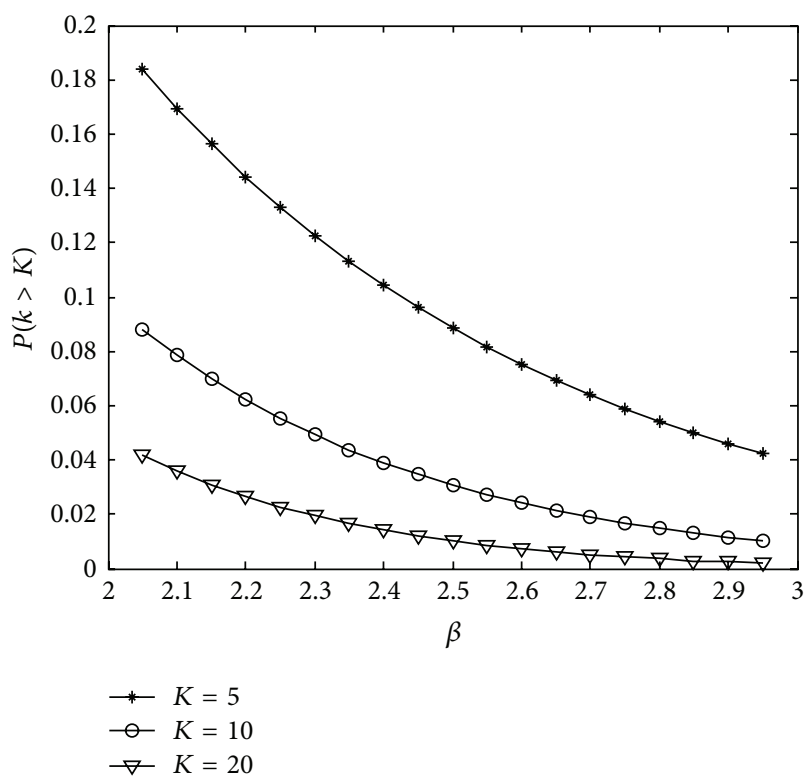

FIGURE 1: The characteristics of cumulative probability with $m=1$ and $N=1000$.

where $m$ and $M$ are the minimal and the maximum degree in finite network. From the literature [16], we can get the maximum degree of scale-free networks:

$$
M \approx m N^{1 /(\beta-1)} .
$$

Substituting (4) into (3), we can obtain

$$
c \approx \frac{1-\beta}{m^{1-\beta}\left(N^{-1}-1\right)} .
$$

According to (2) and (5), we can obtain the density function of degree distribution as follows:

$$
f(k) \approx \frac{(1-\beta) k^{-\beta}}{m^{1-\beta}\left(N^{-1}-1\right)} .
$$

Formula (6) introduces the characteristics of the density function on degree of scale-free networks. We know that the scale-free network is heterogeneous network, and we observe the cumulative probability on degree and investigate the robustness of scale-free networks; formula (7) presents the cumulative probability of scale-free network:

$$
\begin{aligned}
P\{k>K\} & =1-P\{k \leq K\}=1-\int_{m}^{K} f(k) d k \\
& =1-c \int_{m}^{K} k^{-\beta} d k=1-\frac{K^{1-\beta}-m^{1-\beta}}{m^{1-\beta}\left(N^{-1}-1\right)} .
\end{aligned}
$$

Figure 1 introduces the properties of the cumulative probability on degree distribution of the scale-free network, and we can declare that the cumulative probability decreases with the increase of the parameter $\beta$; meanwhile, with the increase of $K$, the cumulative probability decreases. And 


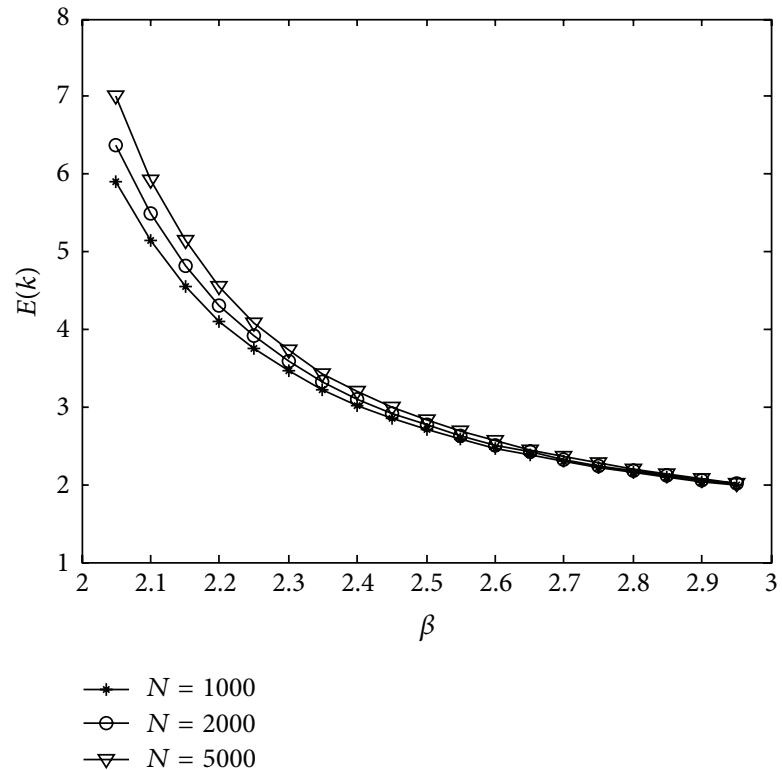

FIGURE 2: The property of average degree versus $\beta$ with different $N$.

we find that the cumulative probability is very small when $K=20$; that is to say, there are few vertices which are degree surpassing $K=20$; this phenomenon reflects the heterogeneity of scale-free networks.

According to (4), (6), and continuous approximation principle, we can obtain the average degree as follows:

$$
\begin{aligned}
E(k) & =\frac{1}{N} \sum_{i=m}^{M} d_{i} \approx \int_{m}^{M} k f(k) d k=c \int_{m}^{M} k^{1-\beta} d k \\
& =\frac{c\left(M^{2-\beta}-m^{2-\beta}\right)}{2-\beta} \\
& =\frac{m(\beta-1)\left(1-N^{(2-\beta) /(\beta-1)}\right)}{(\beta-2)\left(1-N^{-1}\right)} .
\end{aligned}
$$

Figure 2 describes the property of the average degree of scale-free networks, and we discover that the average degree decreases with the increase of the scale exponent $\beta$ and the average degree decreases with the decrease of the number of nodes. From the real case, we know that the larger the average degree is, the better the robustness is, so the smaller the parameter $\beta$ is, the better the robustness is.

\section{Optimal Robustness Design Based on ATND}

A complex network model $G=\langle V, E\rangle, V=\left\{v_{1}, v_{2}, \ldots, v_{N}\right\}$ is the set of nodes, and $E \subseteq V \times V$ is the set of the edges. Meanwhile the adjacent matrix is defined as follows:

$$
H=\left(h_{i j}\right)_{N \times N}, \quad h_{i j}= \begin{cases}1 & \left(v_{i}, v_{j}\right) \in E \\ 0 & \left(v_{i}, v_{j}\right) \notin E .\end{cases}
$$

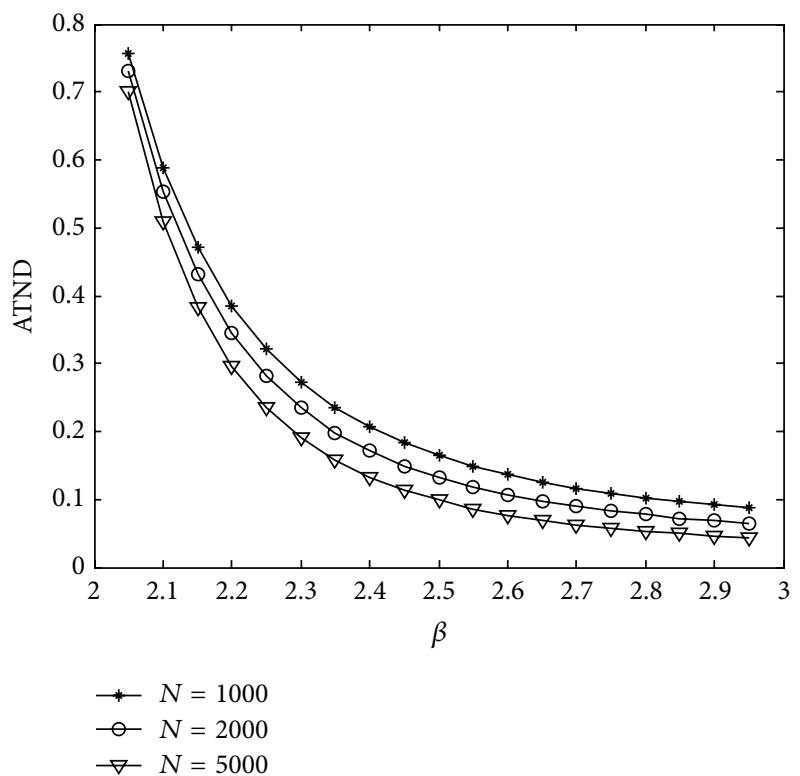

FIgURE 3: The characteristics of ATND versus $\beta$ with different $N$.

Hence, the degree on adjacent nodes of node $v_{i}$ can be obtained by

$$
D_{i}=\sum_{v_{j} \in G_{i}} d_{j}
$$

where $G_{i}=\left\{v_{\omega} \in V \mid v_{i} \in V \wedge\left(v_{\omega}, v_{i}\right) \in E\right\}, i, \omega=1,2, \ldots, N$, and $d_{i}$ is the degree of node $v_{i}$. We calculate the degree of adjacent nodes of networks and we can get the following formula:

$$
D=H d=\sum_{i=1}^{N} D_{i}=\sum_{i=1}^{N} \sum_{v_{j} \in G_{i}} d_{j}=\sum_{i=1}^{N} d_{i}^{2}=\|d\|_{2}^{2},
$$

where $d=\left(d_{1}, d_{2}, \ldots, d_{N}\right)^{T}$ and $D=\left(D_{1}, D_{2}, \ldots, D_{N}\right)^{T}$ are the degree vector and the adjacent degree vector. Based on the above analysis, we define $\sqrt{D}$ as the 2-norm degree of the network; therefore we define the average 2-norm degree (ATND) as follows:

$$
\operatorname{ATND}=\frac{1}{N} \sqrt{D}=\frac{1}{N}\|d\|_{2}=\frac{1}{\sqrt{N}} \sqrt{\frac{1}{N} \sum_{i=1}^{N} d_{i}^{2}} .
$$

According to continuous approximation, we can get the quantitative value of ATND as follows:

$$
\begin{aligned}
\operatorname{ATND} & \approx \frac{1}{\sqrt{N}} \sqrt{\int_{m}^{M} k^{2} f(k) d k} \\
& =\frac{m}{\sqrt{N}} \sqrt{\frac{\beta-1}{3-\beta} \cdot \frac{N^{(3-\beta) /(\beta-1)}-1}{N^{-1}-1}} .
\end{aligned}
$$

From Figure 3, we know that ATND decreases with the increase of the parameter $\beta$, and the smaller the parameter $\beta$ 


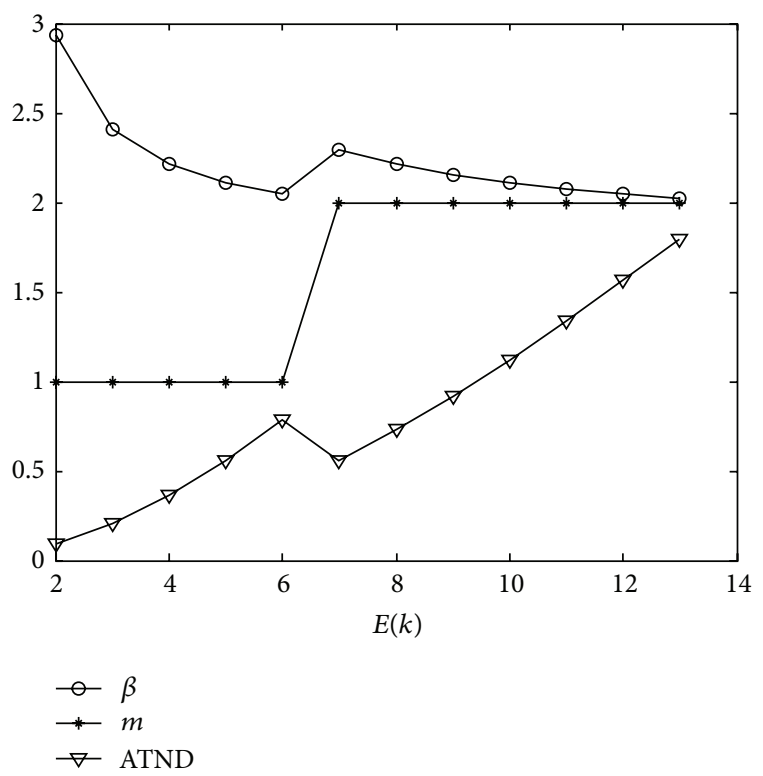

FIgURE 4: The optional value ATND, the optional solutions $\beta$, and $m$ versus $E(k)$.

is, the better the robustness is. Meanwhile, with the increase of the number of nodes, we obtain that ATND decreases which is different from the result given in Figure 2, so we can declare that, with the increase of the number of vertices, the robustness decreases to some extent. So we can declare that the robustness of the scale-free network can be measured by many measurements and different measurements have different results.

Next, we consider the optimal average 2-norm degree; we construct the following nonlinear mixed integer programming scheme:

$$
\begin{array}{ll}
\max & \text { ATND } \\
\text { s.t. } & E(k)=\text { const; } \\
& 2<\beta<3 \\
& 1 \leq m \leq N-1, \\
& m \in Z .
\end{array}
$$

Figure 4 portrays the characteristics of optimal design of scale-free network based on ATND and describes the optimal value ATND and the optional solutions of $\beta$ and $m$ with different average degree $E(k)$ and $N=1000$. From Figure 4, we find that, with the increase of $E(k)$, the minimal degree $m$ increases and the scaling exponent $\beta$ has the behavior of oscillation. Meanwhile, with the increase of the average degree, the optimal average 2-norm degree has the trend to become larger, so the robustness becomes better. Moreover there is an oscillation about ATND proposed in Figure 4, which is because of the fact that the scale-free networks are heterogeneous networks. Furthermore, when $E(k)=7$, these three parameters have the obvious oscillation, which indicates that this point is a tradeoff point between the robustness and cost.

\section{Optimal Robustness Design Based on CV}

In this section, we discuss the robustness of scale-free networks based on CV. From Section 2, we know that the average degree is a robustness measurement of network, but the average degree is a one-sided measurement and it is an overall measure. We know that the larger the cumulative degree is, the larger the average degree is, so the better the robustness is, but the average degree does not portray the relationships between degree and the average degree. Similar to star networks, if the load of one edge exceeds its maximal load, the edge would be collapsing, so there would be an isolated node in this star network. In order to investigate the reliability of networks, we introduce variance degree $[17,18]$ of scale-free networks as follows:

$$
V(k)=E[k-E(k)]^{2},
$$

where $V(\cdot)$ represents the variance, $E(\cdot)$ represents the mathematical expectation, and $k$ is the degree of the node. According to the characteristics of variance, we know that $V(\cdot)$ is the measurement which describes the average distance from the degree $k$ to the average degree of vertices; that is to say, the variance degree describes the relationship between individual measure and overall measure. So we can better comprehend degree distribution according to variance degree: the larger the variance degree is, the worse the degree distribution is; on the contrary, the smaller the variance degree is, the more uniform the degree distribution is, so the better the robustness is. In terms of formulas (4) and (6) and continuous approximation principle, we can estimate the variance degree as follows:

$$
\begin{aligned}
V(k)= & E[k-E(k)]^{2}=E\left[k^{2}\right]-[E(k)]^{2} \\
= & \frac{1}{N} \sum_{k=m}^{M} k^{2}-\left[\frac{1}{N} \sum_{k=m}^{M} k\right]^{2} \\
\approx & \int_{m}^{M} k^{2} f(k) d k-\left[\int_{m}^{M} k f(k) d k\right]^{2} \\
= & \frac{m^{2}(\beta-1)\left(N^{(3-\beta) /(\beta-1)}-1\right)}{(3-\beta)\left(1-N^{-1}\right)} \\
& -\left[\frac{m(\beta-1)\left(1-N^{(2-\beta) /(\beta-1)}\right)}{(\beta-2)\left(1-N^{-1}\right)}\right]^{2} .
\end{aligned}
$$

Formula (16) introduces the characteristics of the variance degree, and we obtain the relationships between variance degree and the parameters of scale-free network. Because the variance degree is the distance from the degree to the average degree, we know that the variance degree is an individual measurement of scale-free network. According to the property of variance, we find that the smaller the variance degree is, the better the robustness is with fixed average degree. From Section 2, we know that the average degree is the overall measurement which neglects the individual 


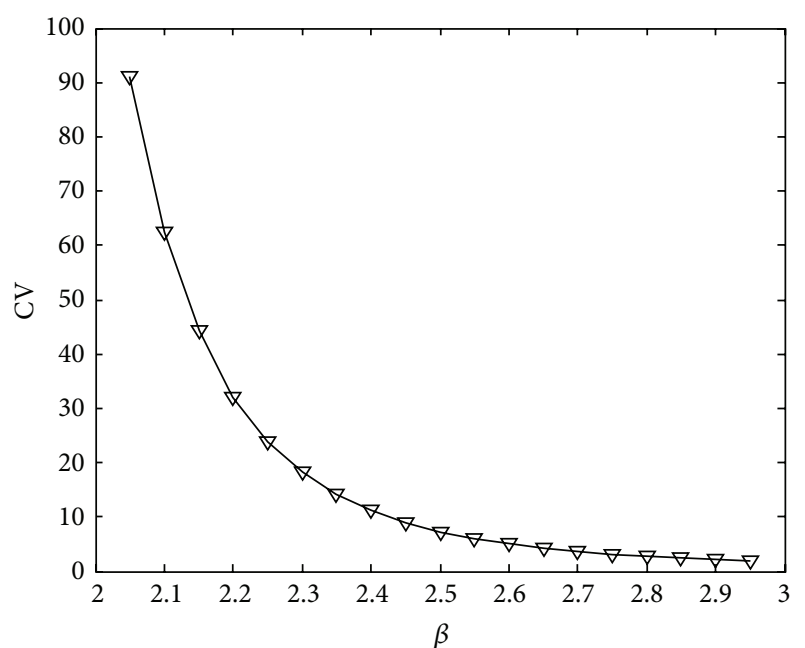

FIGURE 5: Behaviors of the variant coefficient versus scale exponent.

behaviors and the relationships between overall characteristics and individual properties of nodes, so the variance degree is investigated to consider the relationship between them. According to these two aspects, many complex artificial networks would be constructed to suit the needs of society.

From the average degree and the variance degree, we know that the average degree depicts the overall connectivity and the variance degree portrays the individual connectivity, so we must combine these two aspects to construct another better measure which is called CV to investigate the robustness of scale-free network. CV is proportional to the variance degree and inversely proportional to the average degree; it considers not only the overall behaviors of the network but also the individual behaviors of network, so it is a better measure than the average degree and the variance degree. From the definition and the characteristics of the average degree and variance degree, we know that the smaller the $\mathrm{CV}$ is, the better the robustness is of the scale-free network.

Figure 5 portrays the characteristics of $\mathrm{CV}$ and we know that it can reflect the relationships between the average degree and the variance degree versus scaling exponent. CV, which avoids some shortcomings of the average degree and the variance degree,is a better measure to investigate the robustness of networks. If we want to improve the robustness of scale-free network, we must improve the average degree and reduce the variance degree. From Figure 5, we find that $\mathrm{CV}$ decreases with the increase of the scaling exponent $\beta$, and we can tell that, with the decrease of $\mathrm{CV}$, the robustness increases and the resilience to random failure or intentional attacks becomes stronger.

According to the properties of real networks and considering the cost of networks, we design the optimal model to investigate the robustness of scale-free network. From the relationship between the robustness and the heterogeneity and studying the meanings of literatures [12, 13], we design another nonlinear mixed integer programming scheme about

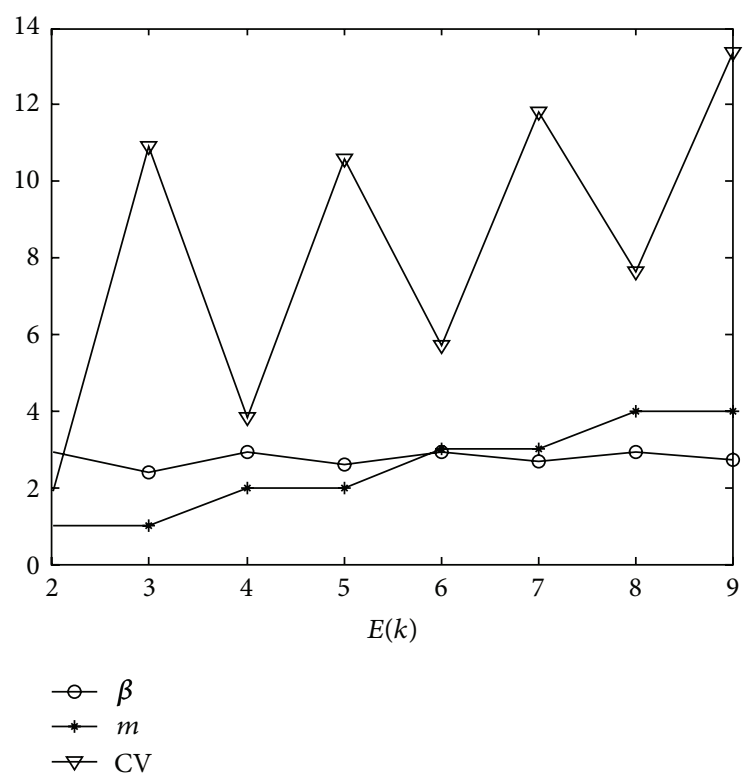

FIgURE 6: The optional value $\mathrm{CV}$, the optional solutions $\beta$, and $m$ versus $E(k)$ with $N=1000$.

$\mathrm{CV}$ to obtain the optional values of all the parameters as follows:

$$
\begin{array}{ll}
\min & \mathrm{CV} \\
\text { s.t. } & E(k)=\text { const; } \\
& 2<\beta<3, \\
& 1 \leq m \leq N-1, \\
& m \in Z .
\end{array}
$$

We portray the characteristics of optimal design (17) in Figure 6, which describes the optimal value $\mathrm{CV}$ and the optional solutions of $\beta$ and $m$ with different $E(k)$. From Figure 6 , we discover that, with the increase of $E(k)$, the minimal degree $m$ increases and the scaling exponent $\beta$ has the behavior of oscillation. Meanwhile, with the increase of $E(k), \mathrm{CV}$ has an obvious oscillation and has the trend to increase. From Figure 6, we can obtain the optimal values of different parameters, and we also can design the real network according to these values to improve the robustness of the real network. Furthermore we find that when $E(k)=2,4,6,8$, $\mathrm{CV}$ has small values and the scale-free networks have the homogenous degree distribution; therefore the networks have the better robustness. In particular when $E(k)=4,6,8$, the simulation results are more to meet the actual situations, and, from Figure 6, we find that when $E(k)=4$, the CV has the smallest value; therefore the scale-free networks have the best robustness among these actual situations.

\section{Discussion of Optimal Robustness}

In the paper, we investigate two optimal models on degree of scale-free network. From our optimal design, we find that different models have different behaviors in measuring the 


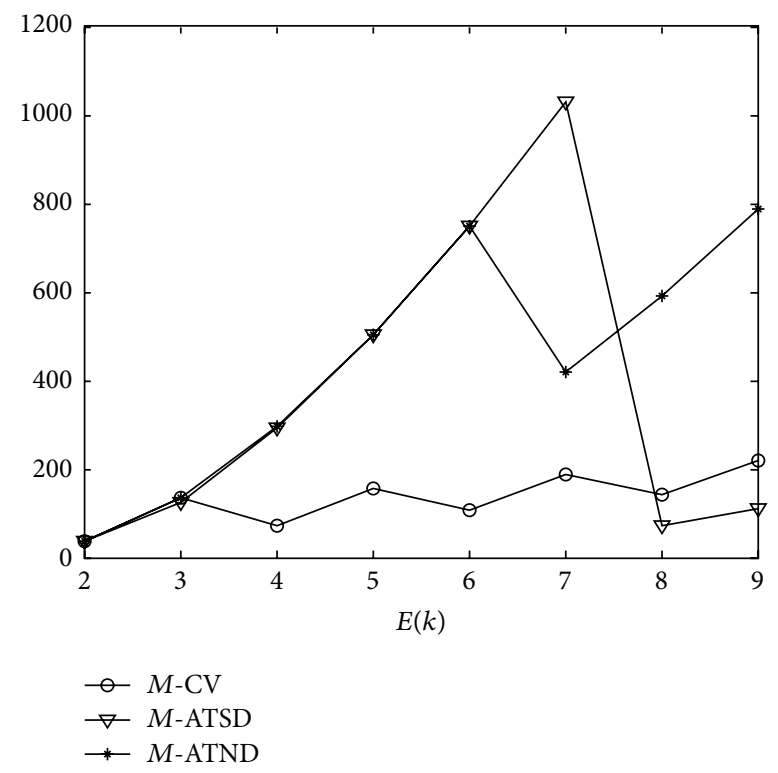

FIgURE 7: The maximum degree of different optimal robustness models versus average degree.

robustness of network, and the robustness can be investigated by the maximum and minimum degree of scale-free network. With the fixed average degree, we find that the smaller the $\mathrm{CV}$ is, the better the robustness is; meanwhile, the smaller the maximum degree is, the more homogeneous the degree distribution is and therefore the better the robustness is; moreover the larger the minimum degree is, the more homogeneous the degree distribution is and the better the robustness is. Hence the maximum and the minimum degree are the other two measures on robustness of scale-free network.

In this section, we discuss the robustness of optimal models according to maximum and minimum degree of scale-free network. With fixed average degree $E(k)$, we know that the robustness becomes better with the decrease of the maximum degree. Figure 7 introduces the characteristics of the maximum degrees of the optimal models versus the average degree. In this section, we use $M-C V, M-A T S D$, and $M$-ATND to represent the maximum degrees of models about optimal CV, ATSD, and ATND, respectively. Figure 7 illustrates that $M$-ATSD is larger than the other two cases with $E(k)$ from 2 to 7 and smaller than the other two cases with $E(k)$ from 8 to 9 . Meanwhile Figure 7 tells us that $M-C V$ has small values all the time, and it has a slight increase. So we can declare that $M-\mathrm{CV}$ among them is the best selection to assess the robustness of scale-free network; that is to say, $\mathrm{CV}$ is a better measure than ATSD and ATND.

Figure 8 gives us another evidence which indicates the fact that CV is a better measure than ATSD and ATND. We use $m$-CV, $m$-ATSD, and $m$-ATND to represent the minimum degrees of optimal models about CV, ATSD, and ATND, respectively. Figure 8 indicates that, with fixed average degree, the minimum degree of optimal ATSD is the smallest degree in these three optimal models and the optimal CV have the largest smallest degree in these three cases. Based on Figures 7 and 8, compared with the largest

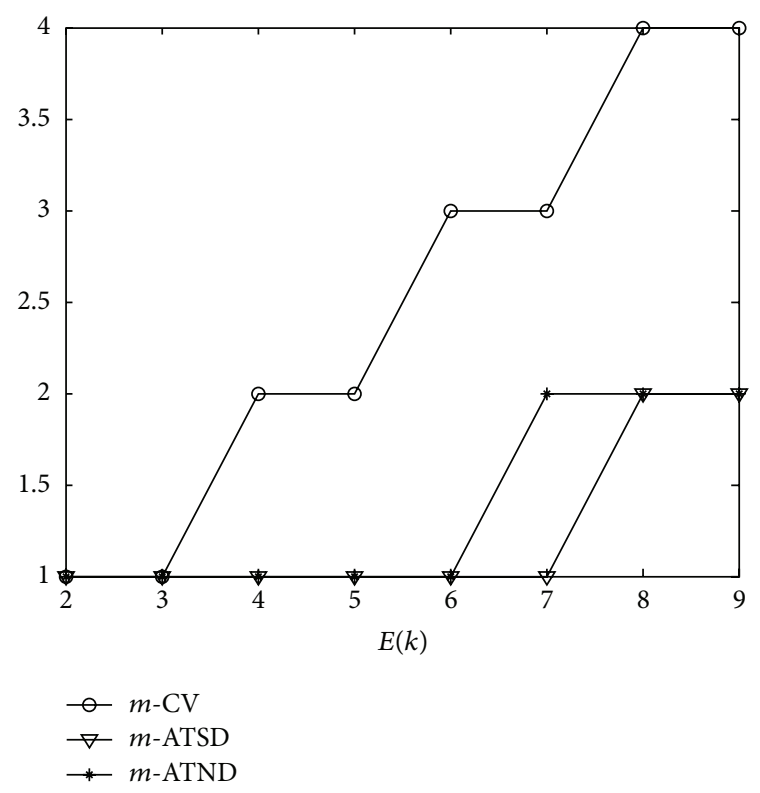

FIGURE 8: The minimum degree of different optimal models versus average degree.

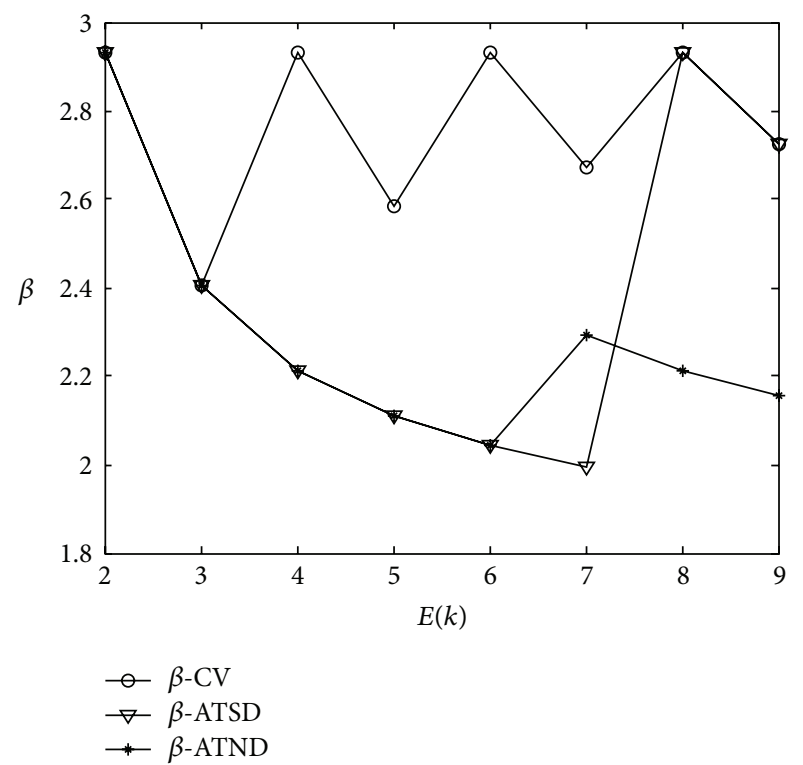

FIGURE 9: The scaling exponent values of different optimal models versus average degree.

degree of minimum degree of optimal ATSD and ATND, with fixed average degree, optimal CV scheme has smaller maximum degree and larger minimum degree; that is to say, the degree distribution of $\mathrm{CV}$ is more homogeneous than that of the other two cases, so we can declare that CV is a better robustness measure than the other two models.

Figure 9 depicts characteristics of the optimal scaling exponent $\beta$ of three different optimal models and Figure 9 shows that the scaling exponent value of optimal CV model is larger than that of the other two optimal models. And in few cases, the optimal scaling exponent values of different models are equal, and, in some cases, they have little differences. 
Meanwhile Figure 9 indicates that the average degree and the two-step degree are very lopsided in investigating the robustness on degree of scale-free network; hence CV is a better model in studying the robustness of scale-free network.

\section{Conclusion}

Complex networks have been paid increasing attention in the past two decades, and many valuable results have been obtained from several aspects $[19,20]$. In this paper, we reestimate the parameter of the density function on degree and discuss the basic behaviors of scale-free network. Based on the density function, we discuss the characteristics of cumulative probability of degree and introduce the properties of the average degree. Meanwhile we give two basic robustness measures of scale-free networks, which are called 2norm degree and $\mathrm{CV}$, and we also construct two nonlinear mixed integer programming schemes according to the average 2-norm degree and CV. Moreover the optimal values of three optimal models can be obtained in this paper and we compare the optimal values of the corresponding parameters of different models. Furthermore we give several evidences of optimal values of optimal designs to illustrate the fact that $\mathrm{CV}$ is a better measure than ATSD and ATND to investigate the robustness of scale-free networks. In the future, we will continue to investigate the robustness and optimal design of scale-free networks; meanwhile we will also investigate the robustness and optimal design of the actual networks.

\section{Competing Interests}

The authors declare that they have no competing interests.

\section{Acknowledgments}

This work was jointly supported by the National Natural Science Foundation of China (61304175, 61503166, 61305149, and 61503164) and the Natural Science Foundation for Youths of Jiangsu Province of China (BK20140241).

\section{References}

[1] S. H. Strogatz, "Exploring complex networks," Nature, vol. 410, no. 6825 , pp. 268-276, 2001.

[2] D. J. Watts and S. H. Strogatz, "Collective dynamics of 'smallworld' networks," Nature, vol. 393, no. 6684, pp. 440-442, 1998.

[3] A.-L. Barabasi and R. Albert, "Emergence of scaling in random networks," Science, vol. 286, no. 5439, pp. 509-512, 1999.

[4] R. Albert and A.-L. Barabasi, "Statistical mechanics of complex networks," Reviews of Modern Physics, vol. 74, no. 1, pp. 47-97, 2002.

[5] R. Albert, H. Jeong, and A. L. Barabasi, "Attack and error tolerance of complex networks," Nature, vol. 406, pp. 378-382, 2000.

[6] S. N. Dorogovtsev and J. F. F. Mendes, "Evolution of networks," Advance in Physics, vol. 51, no. 4, pp. 1079-1187, 2002.

[7] M. E. Newman, "The structure and function of complex networks," SIAM Review, vol. 45, no. 2, pp. 167-256, 2003.
[8] L. Zhao, K. Park, and Y.-C. Lai, "Attack vulnerability of scalefree networks due to cascading breakdown," Physical Review E, vol. 70, no. 3, Article ID 035101, 2004.

[9] J. W. Wang, L. L. Rong, L. Zhang, and Z. Z. Zhang, "Attack vulnerability of scale-free networks due to cascading failures," Physica A, vol. 387, no. 26, pp. 6671-6678, 2008.

[10] S. Boccaletti, V. Latora, Y. Moreno, M. Chavez, and D.-U. Hwang, "Complex networks: structure and dynamics," Physics Reports, vol. 424, no. 4-5, pp. 175-308, 2006.

[11] A. E. Motter and Y.-C. Lai, "Cascade-based attacks on complex networks," Physical Review E, vol. 66, no. 6, Article ID 065102, 2002.

[12] Z.-H. Wu and H.-J. Fang, "Cascading failures of complex networks based on two-step degree," Chinese Physics Letters, vol. 25, no. 10, pp. 3822-3825, 2008.

[13] B. Wang, H. W. Tang, C. H. Guo, and Z. L. Xiu, "Entropy optimization of scale-free networks' robustness to random failures," Physica A, vol. 363, no. 2, pp. 591-596, 2006.

[14] J. Wu, Y.-J. Tan, H.-Z. Deng, and D.-Z. Zhu, "Normalized entropy of rank distribution: a novel measure of heterogeneity of complex networks," Chinese Physics, vol. 16, no. 6, pp. 15761580, 2007.

[15] M. E. J. Newman, S. H. Strogatz, and D. J. Watts, "Random graphs with arbitrary degree distributions and their applications," Physical Review E, vol. 64, no. 2, Article ID 026118, 2001.

[16] R. Cohen, K. Erez, D. Ben-Avraham, and S. Havlin, "Resilience of the Internet to random breakdowns," Physical Review Letters, vol. 85, no. 21, pp. 4626-4628, 2000.

[17] E. Tsukamoto and S. Shirayama, "Influence of the variance of degree distributions on the evolution of cooperation in complex networks," Physica A, vol. 389, no. 3, pp. 577-586, 2010.

[18] T. A. Snijders, “The degree variance: an index of graph heterogeneity," Social Networks, vol. 3, no. 3, pp. 163-174, 1981.

[19] J. Wang, "Mitigation of cascading failures on complex networks," Nonlinear Dynamics, vol. 70, no. 3, pp. 1959-1967, 2012.

[20] J. Wang, "Mitigation strategies on scale-free networks against cascading failures," Physica A: Statistical Mechanics and its Applications, vol. 392, no. 9, pp. 2257-2264, 2013. 

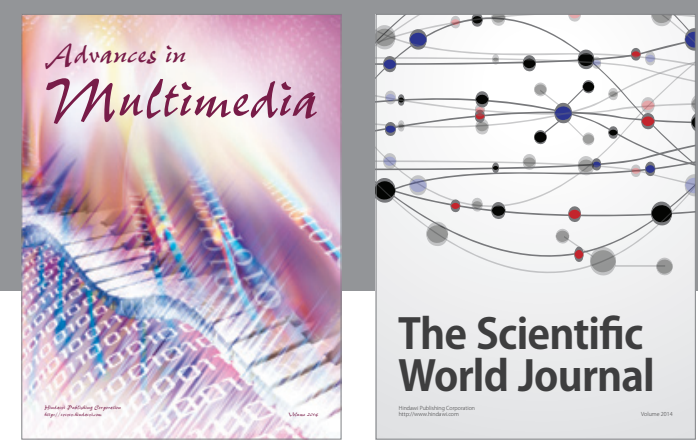

The Scientific World Journal
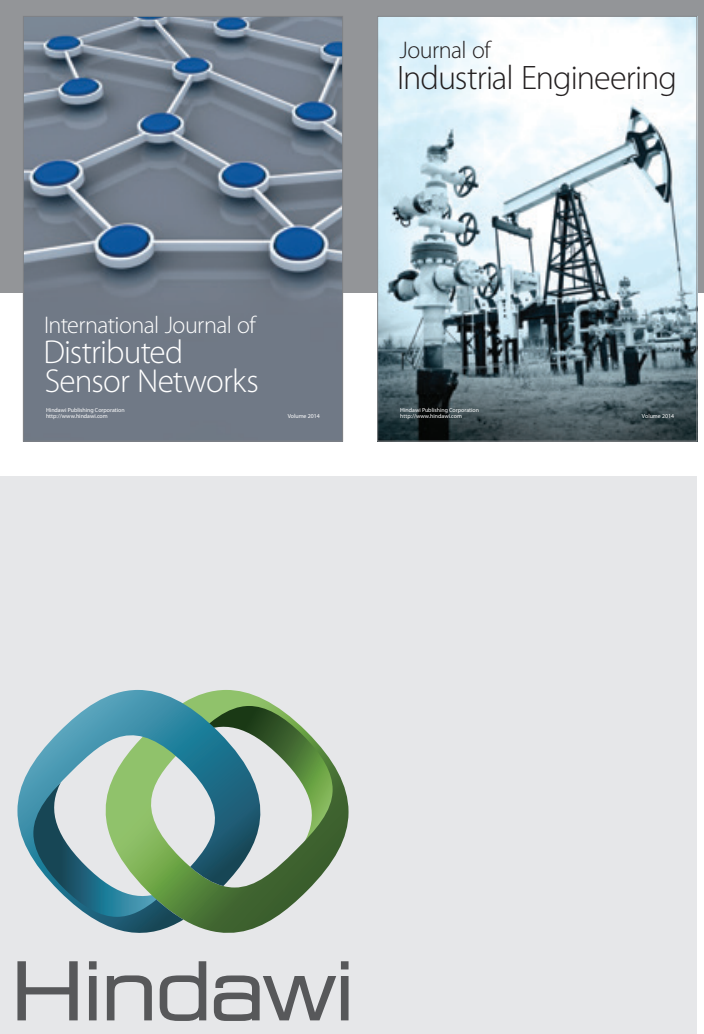

Submit your manuscripts at

http://www.hindawi.com

\section{Computer Networks} and Communications
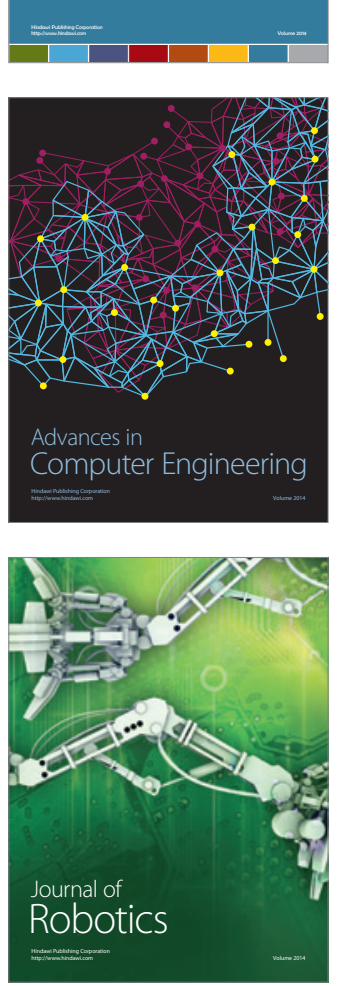
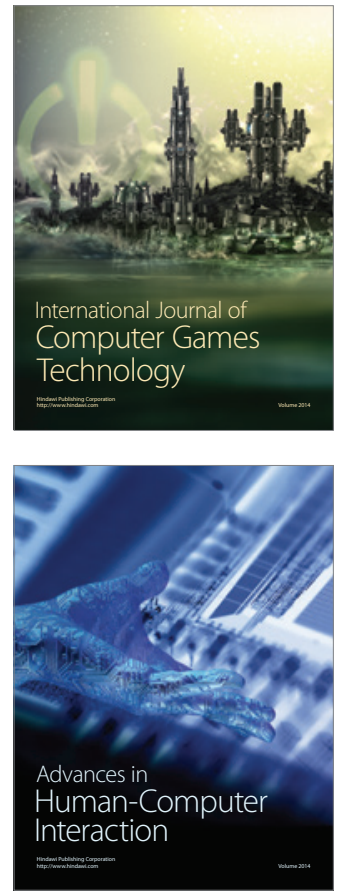
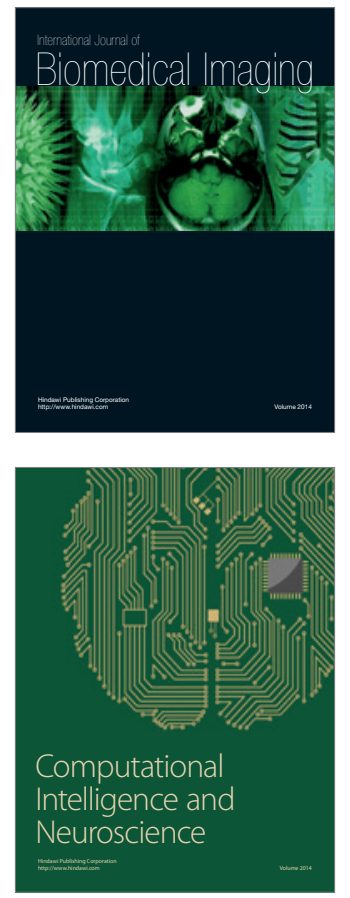
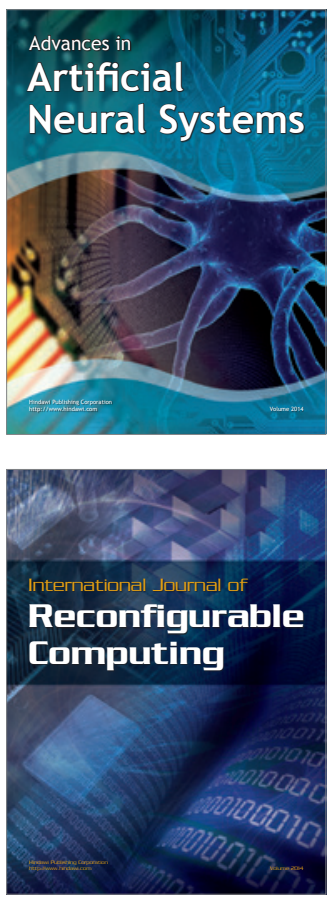
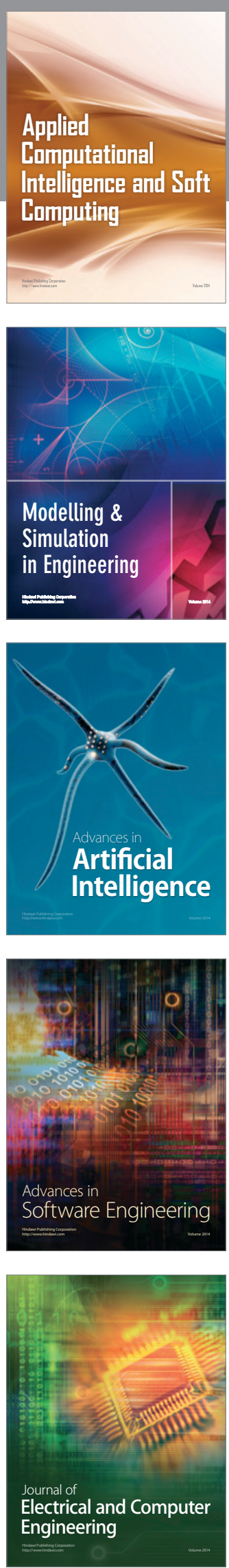